\title{
Facile access to oleuropein and hydroxytyrosol from Ligustrum vulgare - a plant material growing all over Eurasia
}

\author{
Josephine M. Gießel ${ }^{1}$, Anne Zaar ${ }^{1}$, Renate Schäfer ${ }^{1}$, Ahmed Al-Harrasi ${ }^{2}$ and René Csuk ${ }^{1, *}$ \\ ${ }^{1}$ Full Address: Martin-Luther-University Halle-Wittenberg, Organic Chemistry, Kurt-Mothes-Str. 2, D-06120 \\ Halle (Saale), Germany \\ ${ }^{2}$ Full Address: University of Nizwa, Chair of Oman's Medicinal Plants and Marine Natural Products, P.O. Box \\ 33, PC 616, Birkat Al-Mauz, Nizwa, Sultanate of Oman
}

\begin{abstract}
Leaves of Ligustrum vulgare are an alternative, sustainable source for oleuropein. Acidic hydrolysis of oleuropein furnishes hydroxytyrosol in good yield. This approach is one of the most convenient ways to access significant amounts of oleuropein as well as of hydroxytyrosol from plant material readily accessible all over the year and throughout Eurasia. Harvesting the leaves in winter doubles the amount of oleuropein that can be extracted from the plant material.
\end{abstract}

Keywords: Oleuropein; hydroxytyrosol; Ligustrum vulgare; extraction.

\section{Introduction}

In principle, any molecule can be synthesized using sufficient human and material resources. These syntheses are independent of the molecule's size and stereochemical complexity. This has been impressively proven in the past with the synthesis of urea (Wöhler 1828), strychnine (Woodward 1954), vitamin B12 (Woodward and Eschenmoser 1973), and palytoxin (Kishi 1994). All of these syntheses greatly enriched our knowledge of methods and strategies. However, as the example of paclitaxel has shown, (partial)-syntheses are more efficient and above all -more economical than total syntheses. A prerequisite for the latter in particular is the identification of a suitable precursor or of a target molecule to be isolated, for example from microorganisms or plants.
Hydroxytyrosol (1, Scheme 1) is subject to several clinical trials dealing with diseases to evaluate its potential to lower postprandial blood sugar levels in persons suffering from diabetes mellitus ${ }^{1,2}$, or to investigate its influence on blood lipid levels to reduce the risk of several heart diseases ${ }^{3-8}$. Also, it was found ${ }^{9}$ that $\mathbf{1}$ increased the endogenous level of vitamin $\mathrm{C}$, and $\mathbf{1}$ also influenced the progress of inflammation. Hydroxytyrosol also had some effect in the regulation of oxidative stressrelated genes ${ }^{10-12}$. It seems to be responsible for the nutraceutical properties and pharmacological effect of olive oil. Furthermore, adherence to the Mediterranean diet has been associated with a reduced incidence of neurodegenerative diseases, better cognitive performance and a lowered risk of cancer ${ }^{13-15}$.

\section{Results and Discussion}<smiles>C/C=C1/[C@H](CC(=O)OCCc2ccc(O)c(O)c2)OC=C(C(C)=O)[C@@H]1C[C@H]1OC(CO)[C@@H](O)C(O)[C@H]1O</smiles>

Oleuropein (2)<smiles>CCCOc1ccc(CCO)cc1O</smiles>

Hydroxytyrosol (1)

Scheme 1. Extraction of oleuropein (2) from plant material and isolation of hydroxytyrosol (1) by hydrolysis (0.1 M aq. hydrochloric acid, $25^{\circ} \mathrm{C}, 48 \mathrm{~h}, 73 \%$ ). 
Several approaches for the synthesis of $\mathbf{1}$ have been reported. Many of them, however, are lengthy, and their over-all yield is low ${ }^{16-29}$. One of the most rewarding approaches seems the hydrolysis of oleuropein (2). This compound is of its interest as a valuable and bio-renewable building block but also because of its cardio- ${ }^{30-34}$, gastro- ${ }^{35-37}$, neuro${ }^{38-40}$, radio- ${ }^{41}$ and hepatoprotective ${ }^{42-47}$ properties. Its antioxidant activity is due to its orthodiphenolic structure. Furthermore, its anti-cancer ${ }^{48-52}$, antidiabetes ${ }^{48-53}$, anti-obesity activity ${ }^{54}$ gave extended research dealing with this secondary natural product and derivatives thereof another impact.

Oleuropein can be obtained from fresh olives and their leaves. The access to the latter material, however, is limited, and its long-distant transport is expensive. Plants being non-endemic to Western Europe or the US have been suggested as sources for the extraction of $2^{55}$. The cultivation, however, of these plants is often limited to green houses. As a consequence, we were looking for a source of oleuropein and its product of hydrolysis, hydroxytyrosol (1), from plant material that can be grown in large amounts all over Eurasia and the US. One of the most grown plants in Eurasia is Ligustrum vulgare, also known as Common Privet. Its leaves are well known in the Mediterranean historical medicine for their oropharyngeal antiinflammatory effects ${ }^{56}$. Quite recently, extracts from this plant came again in the focus of scientific interest due its anti-inflammatory ${ }^{57-60}$, antiproliferative ${ }^{61,62}$ and anti-lipoxygenase activity ${ }^{63}$. Initial studies showed plants of the genus Ligustrum to contain oleuropein. Thus, we were interested in answering the questions whether this plant material is suited for obtaining oleuropein but also whether leaves harvested at different times (winter or summer) differ regarding the content of oleuropein.

Leaves of Ligustrum vulgare were collected, air dried, and extracted. Chromatographic work-up of the extract gave pure oleuropein (2). As a result, leaves harvested in June held only half of the amount of 2 as compared to leaves having been harvested in November. It can be assumed that the higher content of 2 during winter time might be part of a protection mechanism of the plant against herbivores. Although the content of $\mathbf{2}$ in $L$. vulgare (winter) is approximately half of the amount of $\mathbf{2}$ obtained from the leaves of olives (Olea europaea), L. vulgare is grown in huge amounts all over Eurasia and the US while growing of olives is limited to a relatively small geographical region. Furthermore, olives had a low content of $2(0.1 \%)$, and almost no or an insignificant low amount of oleuropein can be found in the pomace.

Hydroxytyrosol (1) is the phenolic part in oleuropein. The cleavage of oleuropein has been described under a variety of different conditions, such as alkaline hydrolysis as well as hydrolysis by a broad variety of hydrolytic enzymes, viz. esterases and lipases 21, 64-67. Reports describing acidic hydrolysis are scarcely found in literature ${ }^{68}$. As a consequence, we studied the hydrolysis of $\mathbf{2}$ with diluted aqueous hydrochloric acid of $\mathbf{2}$ for 48 hours. Cleavage of $\mathbf{2}$ occurred nicely at ambient temperatures; usual work-up and column chromatography gave target compound $\mathbf{1}$ in $73 \%$ isolated yield.

\section{Conclusion}

To sum up, leaves of L. vulgare can be seen as an alternative, sustainable source for oleuropein. This plant material is more easily obtained than the leaves of olives, and they can be harvested all through the year, although harvesting the leaves in winter doubles the amount of $\mathbf{2}$ that can be extracted from the plant material. Acidic hydrolysis of $\mathbf{2}$ furnishes hydroxytyrosol (1) in good yield. Our approach is one of the most convenient ways to access significant amounts of oleuropein as well as of hydroxytyrosol from plant material readily accessible all over the year and throughout Eurasia.

\section{Experimental}

Melting points were determined with a Büchi melting point apparatus M565 and are uncorrected, NMR spectra were recorded on a Varian spectrometer Unity 500 ( $\delta$ given in ppm, $J$ in $\mathrm{Hz}$ ), mass spectra were obtained on a Finnigan MAT LCQ 7000 (electrospray, voltage $4.1 \mathrm{kV}$, sheath gas nitrogen) instrument. The optical rotations were measured on a Perkin-Elmer polarimeter at $20{ }^{\circ} \mathrm{C}$. Machery-Nagel ALUGRAM XtraSIL pre-coated silica gel $60 \mathrm{~F}_{254}$ plates were used for thin layer chromatography. IR spectra were recorded on a Perkin-Elmer FT-IR spectrometer Spectrum 1000 and wave numbers are expressed in $\mathrm{cm}^{-1}$. The absorption spectra were measured on Perkin Elmer Lambda14 spectrometer. The solvents were dried according to usual procedures. A specimen of $L$. vulgare was deposited at the herbarium of the institute. An authentic sample of $\mathbf{2}$ was obtained from Cayman Chemical.

\section{Oleuropein (2)}

Fresh leaves of Ligustrum vulgare $(2.0 \mathrm{~kg}$, harvested in June) were air-dried, shredded and extracted with methanol ( $5 \times 2 \mathrm{~L}$ ) for one day each. The combined filtrates were evaporated under reduced pressure to a final volume of $250 \mathrm{~mL}$ and extracted with ethyl acetate $(7 \times 200 \mathrm{~mL})$. The solvent was evaporated, and the residue was subjected to column chromatography (silica gel, $\mathrm{CHCl}_{3} / \mathrm{MeOH}, 9: 1 \rightarrow 8: 2$ ) to yield crude 2 (31.2 g) that was subjected to a second chromatographic purification (silica gel, $\mathrm{CHCl}_{3} / \mathrm{MeOH}, 10: 0 \rightarrow$ 9:1 $\rightarrow$ $8: 2$ ) to yield pure 2 ( $4.1 \mathrm{~g}, 0.2 \%$ with respect to the plant material). Extraction of fresh leaves of $L$. vulgare (harvested in November, $2.0 \mathrm{~kg}$ ) under the same conditions gave $2(9.6 \mathrm{~g}, 0.5 \%)$ while the 
extraction of leaves of olives (1 kg leaves; plant obtained from a local garden center, harvested in June) gave $2(6.9 \mathrm{~g}, 0.7 \%)$. Extraction of olives (green, pitted, $1 \mathrm{~kg}$, obtained from a local super market) furnished $2(0.95 \mathrm{~g}, 0.1 \%)$ while the extraction of olive pomace $(1 \mathrm{~kg}$, obtained from Becker \& Karsten UG, Andalusia, Spain) gave 2 (100 mg, 0.01\%). Data for 2: off-white solid; m.p. 87-89 ${ }^{\circ} \mathrm{C}$, m.m.p. 87-89 ${ }^{\circ} \mathrm{C} ; \quad \mathrm{R}_{F}=0.06$ $\left(\mathrm{CHCl}_{3} / \mathrm{MeOH}, 95: 5\right) ; 0.34\left(\mathrm{CHCl}_{3} / \mathrm{MeOH}, 4: 1\right)$; $[\alpha]_{\mathrm{D}}=-160.34^{\circ}\left(c=0.315, \mathrm{CHCl}_{3}\right)$;

IR (KBr): $v=3424 s, 2953 w, 1707 m, 1630 m, 1522 w$, $1442 \mathrm{~m}, 1384 \mathrm{~m}, 1285 \mathrm{~m}, 1193 \mathrm{~m}, 1161 \mathrm{~m}, 1076 \mathrm{sm}^{-1}$; UV-vis $(\mathrm{MeOH}): \lambda_{\max }(\log \varepsilon)=256$ (3.80), 303 (3.46) $\mathrm{nm}$;

${ }^{1} \mathrm{H}$ NMR (500 MHz, DMSO-d $\left.\mathrm{d}_{6}\right): \delta=8.71(d$, $J=20.3 \mathrm{~Hz}, 2 \mathrm{H}, \mathrm{OH}(23,24)), 7.53(s, 1 \mathrm{H}, 11-\mathrm{H})$, $6.65(d, J=8.0 \mathrm{~Hz}, 1 \mathrm{H}, 25-\mathrm{H}), 6.62(d, J=2.1 \mathrm{~Hz}$, $1 \mathrm{H}, 22-\mathrm{H}), 6.49(d d, J=8.0,2.1 \mathrm{~Hz}, 1 \mathrm{H}, 21-\mathrm{H}), 5.98$ $(q d, J=6.8,1.3 \mathrm{~Hz}, 1 \mathrm{H}, 12-\mathrm{H}), 5.88(t, J=1.6 \mathrm{~Hz}$, $1 \mathrm{H}, 7-\mathrm{H}), 4.66(d, J=7.8 \mathrm{~Hz}, 1 \mathrm{H}, 6-\mathrm{H}), 4.08(d t t$, $\left.J=20.7,9.9,7.2 \mathrm{~Hz}, 2 \mathrm{H}, 18-\mathrm{H}_{2}\right), 3.87(d d, J=9.1$, $4.3 \mathrm{~Hz}, 1 \mathrm{H}, 9-\mathrm{H}), 3.69$ (ddd, $J=11.6,6.2,1.9 \mathrm{~Hz}$, $1 \mathrm{H}, 5 \mathrm{~b}-\mathrm{H}), 3.66\left(s, 3 \mathrm{H}, \mathrm{OCH}_{3}\right), 3.47(d d d, J=11.2$, $6.2,4.2 \mathrm{~Hz}, 1 \mathrm{H}, 5 \mathrm{a}-\mathrm{H}), 3.24-3.20(m, 1 \mathrm{H}, 2-\mathrm{H})$, $3.20-3.17(m, 2 \mathrm{H}, 4-\mathrm{H}), 3.13-3.10(m, 1 \mathrm{H}, 1-\mathrm{H})$, $3.10-3.08(m, 1 \mathrm{H}, 3-\mathrm{H}), 2.69(t, J=7.2 \mathrm{~Hz}, 2 \mathrm{H}$, $\left.19-\mathrm{H}_{2}\right), 2.64(d d, J=14.4,4.3 \mathrm{~Hz}, 1 \mathrm{H}, 16 \mathrm{~b}-\mathrm{H}), 2.41$ $(d d, J=14.5,9.2 \mathrm{~Hz}, 1 \mathrm{H}, 16 \mathrm{a}-\mathrm{H})), 1.66(d d, J=7.1$, $1.4 \mathrm{~Hz}, 3 \mathrm{H}, 13-\mathrm{CH}_{3}$ );

13C NMR (125 MHz, DMSO-d 6 ): $\delta=171.1(\mathrm{C}-17)$, 166.6 (C-14), 153.8 (C-11), 145.5 (C-24), 144.2 (C-2323), 129.6 (C-8), 128.8 (C-20), 123.5 (C-12), 120.0 (C-21), 116.6 (C-22), 116.0 (C-25), 108.1 (C-10), 99.5 (C-6), 93.4 (C-7), 77.8 (C-4), 77.0 (C-2), 73.7 (C-1), 70.4 (C-3), 65.5 (C-18), 61.6 $(\mathrm{C}-5), 51.7\left(\mathrm{OCH}_{3}, \mathrm{C}-15\right), 40.5(\mathrm{C}-16), 34.2$ (C-19), 30.6 (C-9), 13.4 (C-13) ppm; ${ }^{1} \mathrm{H}$ NMR (500 MHz, $\left.\left.\mathrm{D}_{2} \mathrm{O}\right): \delta=7.55(s, 1 \mathrm{H}, 11-\mathrm{H})\right), 6.90(d, J=8.1 \mathrm{~Hz}$, $1 \mathrm{H}, 25-\mathrm{H}), 6.85(d, J=2.0 \mathrm{~Hz}, 1 \mathrm{H}, 22-\mathrm{H}), 6.46(d d$, $J=8.1,2.0 \mathrm{~Hz}, 1 \mathrm{H}, 21-\mathrm{H}), 6.10-6.04(m, 1 \mathrm{H}, 12-$ $\mathrm{H}), 5.80-5.78(m, 1 \mathrm{H}, 7-\mathrm{H}), 4.91(d, J=8.0 \mathrm{~Hz}$, $1 \mathrm{H}, 6-\mathrm{H}), 4.36(d d d, J=10.9,7.0,5.5 \mathrm{~Hz}, 1 \mathrm{H}, 18 \mathrm{~b}-$ $\mathrm{H}), 4.24-4.19(m, 1 \mathrm{H}, 18 \mathrm{a}-\mathrm{H}), 4.20-4.15(m, 1 \mathrm{H}$, $9-\mathrm{H}), 3.95-3.92(m, 1 \mathrm{H}, 5 \mathrm{~b}-\mathrm{H}), 3.76(d d, J=12.4$, $5.7 \mathrm{~Hz}, 1 \mathrm{H}, 5 \mathrm{a}-\mathrm{H}), 3.76\left(s, 3 \mathrm{H}, \mathrm{OCH}_{3}\right), 3.61-3.56$ $(m, 1 \mathrm{H}, 2-\mathrm{H}), 3.53(d d d, J=9.8,5.7,2.2 \mathrm{~Hz}, 1 \mathrm{H}$, $4-\mathrm{H}), 3.48$ ( $d d, J=9.6,5.5 \mathrm{~Hz}, 1 \mathrm{H}, 3-\mathrm{H}), 3.46$ (dd, $J$ = 9.7, $4.7 \mathrm{~Hz}, 1 \mathrm{H} 1-\mathrm{H}), 2.89-2.85(\mathrm{~m}, 2 \mathrm{H},(19-\mathrm{H})$, $2.71(d d, J=13.7,5.0 \mathrm{~Hz}, 1 \mathrm{H}, 16 \mathrm{~b}-\mathrm{H}), 2.55(d d$, $J=13.7,8.3 \mathrm{~Hz}, 1 \mathrm{H}, 16 \mathrm{a} .-\mathrm{H}), 1.61(d d, J=7.1,1.3$ $\left.\mathrm{Hz}, 3 \mathrm{H}, 13-\mathrm{CH}_{3}\right) \mathrm{ppm} ;{ }^{13} \mathrm{C}$ NMR $\left(125 \mathrm{MHz}, \mathrm{D}_{2} \mathrm{O}\right)$ : $\delta=174.2(\mathrm{C}-17), 169.1$ (C-14), $154.6(\mathrm{C}-11), 143.9$ (C-24), 142.4 (C-23), 131.2 (C-8), 128.2 (C-20), 125.0 (C-12), 121.2 (C-21), 116.6 (C-22), 116.2 (C-25), 108.0 (C-10), 99.4 (C-6), 94.8 (C-7), 76.3 (C-4), 75.6 (C-2), 72.6 (C-1), 69.4 (C-3), 66.3 (C-18), 60.6 (C-5), $51.9\left(\mathrm{OCH}_{3}, \mathrm{C}-15\right), 39.8$ (C-16), 33.3 (C-19), 30.2 (C-9), 12.5 (C-13) ppm;

ESI-MS $(\mathrm{MeOH}): m / z=539\left(100 \%,[\mathrm{M}-\mathrm{H}]^{-}\right), 575$ $\left(4.8 \%,[\mathrm{M}+\mathrm{Cl}]^{-}\right), 1079\left(79.4 \%,[2 \mathrm{M}-\mathrm{H}]^{-}\right)$;
Analysis calcd for $\mathrm{C}_{25} \mathrm{H}_{32} \mathrm{O}_{13}(540.51)$ : C 55.55, H 5.97; found : C 55.50, H 6.23.

Hydroxytyrosol [1, 2-(3,4-dihydroxyphenyl)-ethanol] To a solution of $2(5.0 \mathrm{~g}, 9.25 \mathrm{mmol})$ in water $(100$ $\mathrm{mL})$, aq. hydrochloric acid $(15 \mathrm{~mL}, 0.1 \mathrm{M})$ was added, and stirring at room temperature was continued for 48 hours. Lyophilization of the reaction mixture followed by column chromatography (silica gel, $\mathrm{CHCl}_{3} / \mathrm{MeOH}, 99: 1 \rightarrow$ 95:5) gave $1(1.04 \mathrm{~g}, 73 \%)$ as a slightly yellowish liquid; $\mathrm{R}_{F}=0.29\left(\mathrm{CHCl}_{3} / \mathrm{MeOH}, 95: 5\right)$;

IR (KBr): $v=3307 m, 2950 w, 1605 m, 1519 s, 1444 m$, $1360 m, 1418 m, 1280 s, 1251 s, 1198 s, 1150 m, 1113 s$, $1010 \mathrm{~s}, 954 \mathrm{~m}, 850 \mathrm{~m} \mathrm{~cm}^{-1}$; UV-vis $\left(\mathrm{H}_{2} \mathrm{O}\right): \lambda_{\max }(\log \varepsilon)$ $=299$ (2.92) nm;

${ }^{1} \mathrm{H}$ NMR $\left(500 \mathrm{MHz}, \mathrm{D}_{2} \mathrm{O}\right): \delta=6.90(d, J=8.1 \mathrm{~Hz}$, $1 \mathrm{H}, 25-\mathrm{H}), 6.85(d, J=2.0 \mathrm{~Hz}, 1 \mathrm{H}, 22-\mathrm{H}, 6.76(d d, J$ $=8.1,2.1 \mathrm{~Hz}, 1 \mathrm{H}, 21-\mathrm{H})), 3.80(t, J=6.7 \mathrm{~Hz}, 2 \mathrm{H}$, $\left.\left.\mathrm{OCH}_{2}\right)\right), 2.76\left(t, J=6.7 \mathrm{~Hz}, 2 \mathrm{H}, \mathrm{CH}_{2}\right) \mathrm{ppm}$;

${ }^{13} \mathrm{C}$ NMR (125 MHz, $\left.\mathrm{D}_{2} \mathrm{O}\right): \delta=143.8(\mathrm{C}-24), 142.2$ (C-23), 131.5 (C-20), 121.2 (C-21), 116.7 (C-22), $116.2(\mathrm{C}-25), 62.6\left(\mathrm{OCH}_{2}\right), 37.0\left(\mathrm{CH}_{2}\right) \mathrm{ppm}$;

ESI-MS $(\mathrm{MeOH}): m / z=153\left(100 \%,[\mathrm{M}-\mathrm{H}]^{-}\right), 199$ $\left(3.2 \%,\left[\mathrm{M}+\mathrm{HCO}_{2}\right]^{-}\right), 307\left(11.1 \%,[2 \mathrm{M}-\mathrm{H}]^{-}\right), 385$ $\left(44.4 \%,[2 \mathrm{M}-\mathrm{H}]^{-}\right), 407\left(14.3 \%,[2 \mathrm{M}-2 \mathrm{H}+\mathrm{Na}]^{-}\right)$; Analysis calcd for $\mathrm{C}_{8} \mathrm{H}_{10} \mathrm{O}_{3}$ (154.16): C 62.33, H 6.54; found: C 62.17, H 6.71.

\section{Acknowledgments}

Many thanks are due to M.Sc. S. Tschullik for experimental help, to Dr. D Ströhl and his team for the NMR spectra and to Dr. R. Kluge for the MSmeasurements. IR- and UV-vis spectra and optical rotations were recorded by Ms V. Simon. The authors like to thank Becker \& Karsten UG (Andalucia, Spain) for the generous donation of olive pomace. Financial support by the "ScienceCampus Halle, WCH" (W13004216 to R.C.) is gratefully acknowledged. The authors declare no conflict of interests.

\section{References}

1 - J. J. Reyes, B. Villanueva, J. A. LopezVillodres, J. P. De La Cruz, L. Romero, M. D. Rodriguez-Perez, G. Rodriguez-Gutierrez, J. Fernandez-Bolanos, J. A. Gonzalez-Correa, Neuroprotective Effect of Hydroxytyrosol in Experimental Diabetes Mellitus, J. Agric. Food Chem. 2017, 65, 4378-4383.

2 - T. A. Wani, F. A. Masoodi, A. Gani, W. N. Baba, N. Rahmanian, R. Akhter, I. A. Wani, M. Ahmad, Olive oil and its principal bioactive compound: Hydroxytyrosol - A review of the recent literature, Trends Food Sci. Technol. 2018, 77, 77-90.

3 - M. Fito, M. Cladellas, R. de la Torre, J. Marti, M. Alcantara, M. Pujadas-Bastardes, J. Marrugat, J. Bruguera, M. C. Lopez-Sabater, J. Vila, M. I. Covas, Antioxidant effect of virgin olive oil in 
patients with stable coronary heart disease: a randomized, crossover, controlled, clinical trial, Atherosclerosis 2005, 181, 149-158.

4 - M. Fito, R. Estruch, J. Salas-Salvado, M. A. Martinez-Gonzalez, F. Aros, J. Vila, D. Corella, O. Diaz, G. Saez, R. de la Torre, M.-T. Mitjavila, M. A. Munoz, R.-M. Lamuela-Raventos, V. Ruiz-Gutierrez, M. Fiol, E. Gomez-Gracia, J. Lapetra, E. Ros, L. Serra-Majem, M.-I. Covas, Effect of the Mediterranean diet on heart failure biomarkers: a randomized sample from the PREDIMED trial, Eur. J. Heart Failure, 2014,16, 543-550.

5 - J. Marrugat, M. I. Covas, M. Fito, H. Schroeder, E. Miro-Casas, E. Gimeno, M. C. Lopez-Sabater, R. De La Torre, M. Farre, Effects of differing phenolic content in dietary olive oils on lipids and LDL oxidation: A randomized controlled trial, Eur. J. Nutr. 2004, 43, 140-147.

6 - A. Pedret, U. Catalan, S. Fernandez-Castillejo, M. Farras, R. M. Valls, L. Rubio, N. Canela, G. Aragones, M. Romeu, O. Castaner, R. de la Torre, M. I. Covas, M. Fito, M. J. Motilva, R. Sola, Impact of virgin olive oil and phenolenriched virgin olive oils on the HDL proteome in hypercholesterolemic subjects: a double blind, randomized, controlled, cross-over clinical trial (VOHF study), PLoS One 2015, 10, e0129160/0129161-e0129160/0129119.

7 - R. Takeda, T. Koike, I. Taniguchi, K. Tanaka, Double-blind placebo-controlled trial of hydroxytyrosol of Olea europaea on pain in gonarthrosis, Phytomedicine 2013, 20, 861-864.

8 - S. Tejada, S. Pinya, M. d. Mar Bibiloni, J. A. Tur, A. Pons, A. Sureda, Cardioprotective Effects of the Polyphenol Hydroxytyrosol from Olive Oil, Curr. Drug Targets 2017, 18, 1477-1486.

9 - E. Lopez-Huertas, J. Fonolla, Hydroxytyrosol supplementation increases vitamin $\mathrm{C}$ levels in vivo. A human volunteer trial, Redox Biol. 2017, 11, 384-389.

10 - B. Braam, M. Langelaar-Makkinje, A. Verkleij, H. Bluyssen, T. Verrips, H.A. Koomans, J. A. Joles, J. A. Post, Anti-oxidant sensitivity of donor age-related gene expression in cultured fibroblasts, Eur. J. Pharmacol. 2006, 542, 154-161.

11 - A. Facchini, S. Cetrullo, S. D'Adamo, F. Flamigni, S. Guidotti, M. Minguzzi, A. Facchini, R. M. Borzi, Hydroxytyrosol prevents increase of osteoarthritis markers in human chondrocytes treated with hydrogen peroxide or growth-related oncogene $\alpha$, PLoS One 2014, 9, e109724.

12 - V. Konstantinidou, O. Khymenets, M. I. Covas, R. de la Torre, D. Munoz-Aguayo, R. Anglada, M. Farre, M. Fito, Time course of changes in the expression of insulin sensitivity-related gens after an acute load of virgin olive oil, OMICS 2009, 13, 431-438.

13 - F. Casamenti, M. Stefani, Olive polyphenols: new promising agents to combat aging- associated neurodegeneration, Expert Rev. Neurother. 2017, 17, 345-358.

14 - M. Debbabi, A. Zarrouk, M. Bezine, W. Meddeb, T. Nury, A. Badreddine, E. M. Karym, R. Sghaier, L. Bretillon, S. Guyot, M. Samadi, M. Cherkaoui-Malki, B. Nasser, M. Mejri, S. Ben-Hammou, M. Hammami, G. Lizard, Comparison of the effects of major fatty acids present in the Mediterranean diet (oleic acid, docosahexaenoic acid) and in hydrogenated oils (elaidic acid) on 7 ketocholesterol-induced oxiapoptophagy in microglial BV-2 cells, Chem. Phys. Lipids 2017, 207, 151-170.

15 - H. Gardener, M. R. Caunca, Mediterranean Diet in Preventing Neurodegenerative Diseases, Curr. Nutr. Rep. 2018, 7, 10-20.

16 - S. Azabou, W. Najjar, A. Ghorbel, S. Sayadi, Mild Photochemical Synthesis of the Antioxidant Hydroxytyrosol via Conversion of Tyrosol, J. Agric. Food Chem. 2007, 55, 4877-4882.

17 - K. Bernath-Levin, J. Shainsky, L. Sigawi, A. Fishman, Directed evolution of nitrobenzene dioxygenase for the synthesis of the antioxidant hydroxytyrosol, Appl. Microbiol. Biotechnol. 2014, 98, 4975-4985.

18 - R. Bernini, E. Mincione, M. Barontini, F. Crisante, Convenient Synthesis of Hydroxytyrosol and Its Lipophilic Derivatives from Tyrosol or Homovanillyl Alcohol, J. Agric. Food Chem. 2008, 56, 8897-8904.

19 - P. Bovicelli, R. Antonioletti, S. Mancini, S. Causio, G. Borioni, S. Ammendola, M. Barontini, Expedient synthesis of hydroxytyrosol and its esters, Synth. Commun. 2007, 37, 4245-4252.

20 - M. Brouk, A. Fishman, Improving process conditions of hydroxytyrosol synthesis by toluene-4-monooxygenase, J. Mol. Catal. B: Enzym. 2012, 84, 121-127.

21 - R. Capasso, A. Evidente, S. Avolio, F. Solla, A Highly Convenient Synthesis of Hydroxytyrosol and Its Recovery from Agricultural Waste Waters, J. Agric. Food Chem. 1999, 47, 1745-1748.

22 - D. Deffieux, P. Gossart, S. Quideau, Facile and sustainable synthesis of the natural antioxidant hydroxytyrosol, Tetrahedron Lett., 2014, 55, 2455-2458.

23 - J. C. Espin, C. Soler-Rivas, E. Cantos, F. A. Tomas-Barberan, H. J. Wichers, Synthesis of the Antioxidant Hydroxytyrosol Using Tyrosinase as Biocatalyst, J. Agric. Food Chem. 2001, 49, 1187-1193.

24 - I. Fernandez-Pastor, A. Fernandez-Hernandez, F. Rivas, A. Martinez, A. Garcia-Granados, A. Parra, Synthesis and Antioxidant Activity of Hydroxytyrosol Alkyl-Carbonate Derivatives, J. Nat. Prod. 2016, 79, 1737-1745. 
25 - P. P. Liebgott, M. Labat, L. Casalot, A. Amouric, J. Lorquin, Bioconversion of tyrosol into hydroxytyrosol and 3,4dihydroxyphenylacetic acid under hypersaline conditions by the new Halomonas sp. strain HTB24, FEMS Microbiol. Lett. 2007, 276, 26-33.

26 - K. Napora-Wijata, K. Robins, A. OsorioLozada, M. Winkler, Whole-Cell Carboxylate Reduction for the Synthesis of 3Hydroxytyrosol, ChemCatChem. 2014, 6, 1089-1095.

27 - G. Piersanti, M. Retini, J. L. Espartero, A. Madrona, G. Zappia, An efficient, economical synthesis of hydroxytyrosol and its protected forms via Baeyer-Villiger oxidation, Tetrahedron Lett. 2011, 52, 4938-4940.

28 - J. Yang, J. X. Yang, F. Zhang, G. Chen, W. Pan, R. Yu, S. Wu, P. Tien, Design, synthesis and biological evaluation of small molecular polyphenols as entry inhibitors against H5N1, Bioorg. Med. Chem. Lett. 2014, 24, 2680-2684.

29 - Z. L. Zhang, J. Chen, Q. Xu, C. Rao, C. Qiao, Efficient Synthesis of Hydroxytyrosol from 3,4-Dihydroxybenzaldehyde, Synth. Commun. 2012, 42, 794-798.

30 - I. Andreadou, D. Benaki, P. Efentakis, S. I. Bibli, A.-I. Milioni, A. Papachristodoulou, A. Zoga, A. L. Skaltsounis, E. Mikros, E. K. Iliodromitis, The Natural Olive Constituent Oleuropein Induces Nutritional Cardioprotection in Normal and CholesterolFed Rabbits: Comparison with Preconditioning, Planta Med. 2015, 81, 655-663.

31 - F. Hadrich, A. Mahmoudi, Z. Bouallagui, I. Feki, H. Isoda, B. Feve, S. Sayadi, Evaluation of hypocholesterolemic effect of oleuropein in cholesterol-fed rats, Chem.-Biol. Interact., 2016, 252, 54-60.

32 - Z. Janahmadi, A. A. Nekooeian, A. R. Moaref, M. Emamghoreishi, Oleuropein Offers Cardioprotection in Rats with Acute Myocardial Infarction, Cardiovasc. Toxicol. 2015, 15, 61-68.

33 - A. A. Nekooeian, A. Khalili, M. B. Khosravi, Oleuropein offers cardioprotection in rats with simultaneous type 2 diabetes and renal hypertension, Indian J. Pharmacol. 2014, 46, 398-403.

34 - S. H. Omar, Cardioprotective and neuroprotective roles of oleuropein in olive, Saudi Pharm. J. 2010, 18, 111-121.

35 - S. Al-Quraishy, M.S. Othman, M. A. Dkhil, A. E. Abdel Moneim, Olive (Olea europaea) leaf methanolic extract prevents $\mathrm{HCl} /$ ethanolinduced gastritis in rats by attenuating inflammation and augmenting antioxidant enzyme activities, Biomed. Pharmacother. 2017, 91, 338-349.

36 - I. Hassen, H. Casabianca, K. Hosni, Biological activities of the natural antioxidant oleuropein:
Exceeding the expectation - A mini-review, J. Funct. Foods 2015, 18, 926-940.

37 - S. H. Omar, Olive: native of Mediterranean region and health benefits, Pharmacogn. Rev. 2008, 2, 135-142.

38 - A. R. Khalatbary, H. Ahmadvand, Neuroprotective effect of oleuropein following spinal cord injury in rats, Neurol. Res. 2012, 34, 44-51.

39 - S. Pourkhodadad, M. Alirezaei, M. Moghaddasi, H. Ahmadvand, M. Karami, B. Delfan, Z. Khanipour, Neuroprotective effects of oleuropein against cognitive dysfunction induced by colchicine in hippocampal CA1 area in rats, J. Physiol. Sci., 2016, 66, 397-405.

40 - H. Yu, P. Liu, H. Tang, J. Jing, X. Lv, L. Chen, L. Jiang, J. Xu, J. Li, Oleuropein, a natural extract from plants, offers neuroprotection in focal cerebral ischemia/reperfusion injury in mice, Eur. J. Pharmacol. 2016, 775, 113-119.

41 - O. Benavente-Garcia, J. Castillo, J. Lorente, M. Alcaraz, Radioprotective effects in vivo of phenolics extracted from Olea europaea L. leaves against $\mathrm{X}$-ray-induced chromosomal damage: comparative study versus several flavonoids and sulfur-containing compounds, J. Med. Food 2002, 5, 125-135.

42 - R. Domitrovic, H. Jakovac, V. V. Marchesi, I. Sain, Z. Romic, D. Rahelic, Preventive and therapeutic effects of oleuropein against carbon tetrachloride-induced liver damage in mice, Pharmacol. Res. 2012, 65, 451-464.

43 - A. M. Hegazy, A. S. Abdel-Azeem, H. M. Zeidan, K. S. Ibrahim, E. M. el Sayed, Hypolipidemic and hepatoprotective activities of rosemary and thyme in gentamicin-treated rats, Hum. Exp. Toxicol. 2018, 37, 420-430.

44 - W. Hur, S. W. Kim, Y. K. Lee, J. E. Choi, S. W. Hong, M. J. Song, S. H. Bae, T. Park, S. J. Um, S. K. Yoon, Oleuropein reduces free fatty acid-induced lipogenesis via lowered extracellular signal-regulated kinase activation in hepatocytes, Nutr. Res. 2012, 32, 778-786.

45 - S. W. Kim, W. Hur, T. Z. Li, Y. K. Lee, J. E. Choi, S. W. Hong, K.-S. Lyoo, C. R. You, E. S. Jung, C. K. Jung, T. Park, S. J. Um, S. K. Yoon, Oleuropein prevents the progression of steatohepatitis to hepatic fibrosis induced by a high-fat diet in mice, Exp. Mol. Med. 2014, 46, e92.

46 - Y. Kim, Y. Choi, T. Park, Hepatoprotective effect of oleuropein in mice: mechanisms uncovered by gene expression profiling, Biotechnol. J. 2010, 5, 950-960.

47 - A. Mahmoudi, F. Hadrich, I. Feki, H. Ghorbel, Z. Bouallagui, R. Marrekchi, H. Fourati, S. Sayadi, Oleuropein and hydroxytyrosol rich extracts from olive leaves attenuate liver injury and lipid metabolism disturbance in bisphenol A-treated rats, Food Funct. 2018, 9, 3220-3234.

48 - J.-S. Cheng, C. T. Chou, Y. Y. Liu, W. C. Sun, P. Shieh, D. H. Kuo, C. C. Kuo, C. R. Jan, 
W. Z. Liang, The effect of oleuropein from olive leaf (Olea europaea) extract on $\mathrm{Ca} 2+$ homeostasis, cytotoxicity, cell cycle distribution and ROS signaling in HepG2 human hepatoma cells, Food Chem. Toxicol. 2016, 91, 151-166.

49 - M. H. Elamin, S. S. Al-Maliki, Leishmanicidal and apoptotic activities of oleuropein on Leishmania major, Int. J. Clin. Pharmacol. Ther. 2014, 52, 880-888.

50 - M. Leri, R. Oropesa-Nunez, C. Canale, S. Raimondi, S. Giorgetti, E. Bruzzone, V. Bellotti, M. Stefani, M. Bucciantini, Oleuropein aglycone: A polyphenol with different targets against amyloid toxicity, Biochim. Biophys. Acta, Gen. Subj. 2018, 1862, 1432-1442.

51 - A. Papachristodoulou, M. Tsoukala, D. Benaki, S. Kostidis, K. Gioti, N. Aligiannis, H. Pratsinis, D. Kletsas, A.-L. Skaltsounis, E. Mikros, R. Tenta, Oleuropein is a powerful sensitizer of doxorubicin-mediated killing of prostate cancer cells and exerts its action via induction of autophagy, J. Cancer Res. Treat. 2016, 4, 61-68.

52 - P. Samara, N. Christoforidou, C. Lemus, A. Argyropoulou, K. Ioannou,

K. Vougogiannopoulou, N. Aligiannis, E. Paronis, N. Gaboriaud-Kolar, O. Tsitsilonis, A.-L. Skaltsounis, New semi-synthetic analogs of oleuropein show improved anticancer activity in vitro and in vivo, Eur. J. Med. Chem. 2017, 137, 11-29.

53 - K. J. Park, W. S. Suh, L. Subedi, S. Y. Kim, S. U. Choi, K. R. Lee, Secoiridoid glucosides from the twigs of Syringa oblata var. dilatata and their neuroprotective and cytotoxic activities, Chem. Pharm. Bull. 2017, 65, 359-364.

54 - A. Casado-Diaz, J. Anter, S. Muller, P. Winter, J. M. Quesada-Gomez, G. Dorado, Transcriptomic analyses of the anti-adipogenic effects of oleuropein in human mesenchymal stem cells, Food Funct. 2017, 8 , 1254-1270.

55 - M. Scognamiglio, B. D’Abrosca, V. Fiumano, M. Golino, A. Esposito, A. Fiorentino, Seasonal phytochemical changes in Phillyrea angustifolia L.: Metabolomic analysis and phytotoxicity assessment, Phytochemistry Lett. 2014, 8, 163-170.

56 - A. Pieroni, P. Pachaly, An ethnopharmacological study on common privet (Ligustrum vulgare) and phillyrea (Phillyrea latifolia), Fitoterapia 2000, 71, S89-S94.

57 - E. Giner, M. C. Recio, J. L. Rios, R. M. Giner, Oleuropein Protects against Dextran Sodium Sulfate-Induced Chronic Colitis in Mice, J. Nat. Prod. 2013, 76, 1113-1120.

58 - Y. H. Kim, Y. J. Choi, M. K. Kang, E. J. Lee, D. Y. Kim, H. Oh, Y. H. Kang, Oleuropein
Curtails Pulmonary Inflammation and Tissue Destruction in Models of Experimental Asthma and Emphysema, J. Agric. Food Chem. 2018, 66, 7643-7654.

59 - J. Park, J. S. Min, U. Chae, J. Y. Lee, K. S. Song, H. S. Lee, H. J. Lee, S. R. Lee, D. S. Lee, Anti-inflammatory effect of oleuropein on microglia through regulation of Drp1dependent mitochondrial fission, J. Neuroimmunol. 2017, 306, 46-52.

60 - S.-J. Ryu, H. S. Choi, K. Y. Yoon, O. H. Lee, K. J. Kim, B. Y. Lee, Oleuropein Suppresses LPS-Induced Inflammatory Responses in RAW 264.7 Cell and Zebrafish, J. Agric. Food Chem. 2015, 63, 2098-2105.

61 - S. Bulotta, R. Corradino, M. Celano, M. D'Agostino, J. Maiuolo, M. Oliverio, A. Procopio, M. Iannone, D. Rotiroti, D. Russo, Antiproliferative and antioxidant effects on breast cancer cells of oleuropein and its semisynthetic peracetylated derivatives, Food Chem. 2011, 127, 1609-1614.

62 - J. Han, T. P. N. Talorete, P. Yamada, H. Isoda, Anti-proliferative and apoptotic effects of oleuropein and hydroxytyrosol on human breast cancer MCF-7 cells, Cytotechnology 2009, 59, 45-53.

63 - K. Vougogiannopoulou, C. Lemus, M. Halabalaki, C. Pergola, O. Werz, A.B. Smith, III, S. Michel, L. Skaltsounis, B. Deguin, OneStep Semisynthesis of Oleacein and the Determination as a 5-Lipoxygenase Inhibitor, J. Nat. Prod. 2014, 77, 441-445.

64 - A. D. Bianco, I. Muzzalupo, A. Piperno, G. Romeo, N. Uccella, Bioactive derivatives of oleuropein from olive fruits, J. Agr. Food Chem. 1999, 47, 3531-3534.

65 - A. D. Bianco, A. Piperno, G. Romeo, N. Uccella, NMR experiments of oleuropein biomimetic hydrolysis, J. Agr. Food. Chem. 1999, 47, 3665-3668.

66 - R. Briante, F. La Cara, F. Febbraio, R. Barone, G. Piccialli, R. Carolla, P. Mainolfi, L. De Napoli, M. Patumi, G. Fontanazza, R. Nucci, Hydrolysis of oleuropein by recombinant betaglycosidase from hyperthermophilic archaeon Sulfolobus solfataricus immobilised on chitosan matrix, J. Biotechnol. 2000, 77, 275-286.

67 - R. Briante, F. La Cara, M. P. Tonziello, F. Febbraio, R. Nucci, Antioxidant activity of the main bioactive derivatives from oleuropein hydrolysis by hyperthermophilic betaglycosidase, J. Agr. Food Chem. 2001, 49, 3198-3203.

68 - F. A. Mackellar, R. C. Kelly, E. E. Van Tamelen, C. Dorschel, Structure and Stereochemistry of Elenolic Acid, J. Am. Chem. Soc. 1973, 95, 7155 